\title{
Expression of the Bcl-2 family genes and complexes involved in the mitochondrial transport in prostate cancer cells
}

\author{
ASMARINAH $^{1 *}$, AGNIESZKA PARADOWSKA-DOGAN $^{2 *}$, RIA KODARIAH $^{3}$, BUDIANA TANUHARDJA $^{3}$, \\ PRZEMYSLAW WALISZEWSKI ${ }^{2}$, CHAIDIR ARIF MOCHTAR $^{4}$, YULI BUDININGSIH $^{5}$, \\ WOLFGANG WEIDNER ${ }^{2}$ and ELVIRA HINSCH ${ }^{2}$ \\ ${ }^{1}$ Department of Medical Biology, Faculty of Medicine, University of Indonesia, Jakarta Pusat, Jakarta 10430, Indonesia; \\ ${ }^{2}$ Department of Urology, Pediatric Urology and Andrology, Faculty of Medicine, D-35392 Giessen, Germany; \\ ${ }^{3}$ Department of Pathological Anatomy, Faculty of Medicine, University of Indonesia; ${ }^{4}$ Department of Urology, \\ Faculty of Medicine, University of Indonesia, Cipto Mangunkusumo Hospital; ${ }^{5}$ Department of Forensic Medicine, \\ Faculty of Medicine, University of Indonesia, Jakarta Pusat, Jakarta 10430, Indonesia
}

Received March 4, 2014; Accepted June 19, 2014

DOI: 10.3892/ijo.2014.2576

\begin{abstract}
Alteration of molecular pathways triggering apoptosis gives raise to various pathological tissue processes, such as tumorigenesis. The mitochondrial pathway is regulated by both the genes of the Bcl-2 family and the genes encoding mitochondrial transport molecules. Those proteins allow a release of cyctochrome $c$ through the outer mitochondrial membrane. This release activates the caspase cascade resulting in death of cells. There are at least two main transport systems associated with the family of Bcl-2 proteins that are involved in transport of molecules through the outer mitochondrial membrane, i.e., the voltage dependent anion channels (VDACs) and translocases of the outer mitochondrial membrane proteins (TOMs). We investigated the expression of genes of the Bcl-2 family, i.e., pro-apoptotic Bak and Bid, and anti-apoptotic Bcl-2; VDAC gene, i.e., VDAC1, VDAC2 and VDAC3; and TOMM genes, i.e., TOMM20, TOMM22 and TOMM40. This study was performed at the mRNA and the protein level. Fourteen paraffin embedded prostate cancer tissues and five normal prostate tissues were analyzed by the quantitative PCR array and immunohistochemistry. We found a significant increase in both mRNA expression of the anti-apoptotic $\mathrm{Bcl}-2$ gene and VDAC1 gene in prostate cancer tissue in comparison with their normal counterparts. Translation of the anti-apoptotic
\end{abstract}

Correspondence to: Dr Asmarinah, Department of Medical Biology, Faculty of Medicine, University of Indonesia, Jl. Salemba Raya No. 6, Jakarta Pusat, Jakarta 10430, Indonesia

E-mail: asmarinah.si@gmx.de

*Contributed equally

Key words: Bcl-2 family, voltage dependent anion channels, translocases of the outer mitochondrial membrane expression, prostate cancer
Bcl-2 and VDAC1 genes in prostate cancer tissue was slightly increased. We observed no significant differences in the mRNA expression of the pro-apoptotic Bak and Bid genes, VDAC2 or VDAC3 genes or the three TOMM genes in these tissues. The pro-apoptotic Bax protein was downtranslated significantly in secretory cells of prostate cancer as compared to normal prostate. We suggest that this protein is a good candidate as biomarker for prostate cancer.

\section{Introduction}

Apoptosis (programmed cell death) plays an important role in a variety of biological phenomena including tissue homeostasis, morphogenesis and tumorigenesis $(1,2)$. There are two common pathways for initiation of apoptosis, i.e., the intrinsic (mitochondrial) and extrinsic (or death receptor) pathways. The intrinsic pathway is closely regulated by proteins of the Bcl-2 family. This family consists of two main groups, namely the pro-apoptotic proteins, i.e., Bak, Bax, Bad, Bcl-Xs, Bid, Bik, Bim and Hrk and the anti-apoptotic proteins, i.e., Bcl-2, Bcl-xL, Bcl-W, Bfl-1 and Mcl-1. The anti-apoptotic proteins regulate apoptosis by blocking the release of some mitochondrial molecules, such as cytochrome $c$, whereas the pro-apoptotic proteins promote their release from mitochondria to the cytoplasm. Caspases are activated by cytochrome $c$ in the cytoplasm $(3,4)$.

Alteration of the apoptotic pathways results in a variety of pathological tissue processes including cancer, autoimmune disorders and neurodegenerative diseases. In cancer, there is a loss of balance between cell division and cell death that seems to play a pivotal role in carcinogenesis $(2,5,6)$. It has been reported that the expression of the anti-apoptotic Bcl-2 gene is increased in prostate cancer cells and is associated with a hormone-insensitive, metastatic phenotype of prostate cancer (7-9). The high expression of Bcl-2 protein in surgical specimens of prostate cancer was demonstrated in response to androgen deprivation therapy (ADT) applied prior to radical prostatectomy (10). Whereas the expression of the anti-apop- 
totic Bcl-2 protein was significantly increased in prostate cancer tissues along the increasing Gleason score, expression of the pro-apoptotic Bak and Bax molecules remained similar in these tissues (11).

The anti-apoptotic Bcl-2 and Bcl-x proteins can interact structurally with the voltage dependent anion channel (VDAC), especially with the N-terminal region of VDAC isoform 1 (12). The VDACs are found mostly in the outer mitochondrial membrane (OMM), which are active during the movement of various substances into and out of the mitochondria (reviewed in ref. 13). The VDACs play an essential role in the release of cytochrome $c$ during the apoptotic process (14-16). Moreover, some publications reported that the family of the apoptotic Bcl-2 proteins interact the outer mitochondrial membrane protein (TOM) $(17,18)$. Protein complexes such as TOM and VDAC were proposed to act similarly to Bax receptors in regulation of apoptosis (18). Bellot et al (17) demonstrated that TOM is a receptor for the pro-apoptotic Bax protein. Expression of TOMM22 gene resulted in inhibition of the association between Bax and mitochondria preventing Bax-dependent apoptosis (17). It remains unclear, how VDACs or TOMs interact with the other apoptotic proteins, such as Bax, Bak and $\mathrm{Bcl}-2$ molecules to induce apoptosis. However, the latter one is inhibited during prostate tumorigenesis.

In this context, we investigated both the level of expression of protein complexes and their association with the expression of Bcl-2 family in prostate cancer tissue. We measured the transcript (mRNA) expression level of three members of the Bcl-2 family, i.e., the pro-apoptotic Bak and Bid protein, as well as anti-apoptotic $\mathrm{Bcl}-2$ protein; three isoforms of VDAC, i.e., VDAC1, VDAC2 and VDAC3; and three member genes of TOM complexes, i.e., TOMM20, TOMM22 and TOMM40 in prostate cancer or normal prostate tissues. Based on the transcript expression level, we evaluated in the same tissues the expression level of the family of Bcl-2 proteins, i.e., anti-apoptotic Bcl-2 and pro-apoptotic Bax, as well as outer mitochondrial membrane protein of VDAC1.

\section{Materials and methods}

Samples. We analyzed two groups of tissues, i.e., prostate cancers with Gleason score 6 to 10 and normal prostates. Fourteen paraffin-embedded prostate cancers classified as patterns Gleason 6 to 10. All of them were obtained from the Tissue Archives at the Department of Anatomical Pathology, Faculty of Medicine, University of Indonesia during a period of 2007-2010. These samples were obtained from the prostate cancer patients who underwent prostatectomy. Five normal prostates were obtained from adult human male cadavers at Department of Forensic Medicine, Faculty of Medicine, University of Indonesia using the following criteria: tissues were taken from the deceased within 0 to $12 \mathrm{~h}$ from death, age between 15-25 years, who had no health problems including hypogonadism. A pathologist consultant verified the diagnosis of prostate cancer in the appropriate tissues as well as normal prostate tissue structure in the control group. The study was approved by the local ethics committee and informs consent was obtained from the patients or the family of the deceased.
Tissue processing, RNA extraction and cDNA synthesis. Tumor areas were identified in the $4 \mu \mathrm{m}$-thick, formalin-fixed and paraffin-embedded prostate sections. Normal prostate tissue were put into fixative, embedded in paraffin and cut into $4 \mu \mathrm{m}$-sections for routine histology examination. Total RNA from both kinds of tissues was extracted using High Pure RNA Paraffin Kit (Roche, Mannheim, Germany) according to the manufacturer's protocol. In the protocol, DNase and Proteinase $\mathrm{K}$ were added to improve RNA quality. Concentration of total RNA in the samples was quantified. RNA purity was determined using Nanodrop (Thermo Scientific, Langenselbold, Germany). Subsequently, integrity of RNA was evaluated using Experion (Bio-Rad, Munich, Germany). cDNA was synthesized from $500 \mathrm{ng}$ of total RNA using the RT2 Formalin Fix Paraffin-Embedded (FFPE) PreAMP cDNA synthesis kit (SABiosciences, MD, USA).

PCR array. Quantitative mRNA expressions of three members of the family of Bcl-2 genes, i.e., Bak, Bid and $\mathrm{Bcl}-2$; three isoforms of VDAC, i.e., VDAC1, VDAC2 and VDAC3; and three member genes of TOM complexes, i.e., TOMM20, TOMM22 and TOMM40 were analysed in custom designed PCR array plate for human genes according to the manufacturer's protocol (SABiosciences). As a control, expression of three housekeeping genes GAPDH, ACTB and HPRT1 were investigated in this study. The reaction was performed using commercially available RT2 Nano PreAMP cDNA Synthesis Primer Mix for Human Custom PCR array (SABiosciences). The following conditions were applied: $95^{\circ} \mathrm{C}$ for $10 \mathrm{~min}, 8$ cycles of $95^{\circ} \mathrm{C}$ for $15 \mathrm{sec}, 60^{\circ} \mathrm{C}$ for 2 min. The cDNA was amplified by RT2 Real Time Master Mix SYBR-Green (SABiosciences) using iCycler (Bio-Rad) under the following conditions: $95^{\circ} \mathrm{C}$ for $10 \mathrm{~min}, 40$ cycles of $95^{\circ} \mathrm{C}$ for $15 \mathrm{sec}, 60^{\circ} \mathrm{C}$ for $60 \mathrm{sec}$ and $72^{\circ} \mathrm{C}$ for $20 \mathrm{sec}$.

Immunohistochemical staining of the Bcl-2, Bax and VDAC1 proteins. Based on the mRNA expression results, the expression of the anti-apoptotic Bcl-2 and VDAC1 proteins in both kinds of tissues were evaluated. For the expression in pro-apoptotic protein level, we evaluated pro-apoptotic Bax, instead of the Bak molecule. The formalin fixed paraffinembedded tissue of prostate cancer and normal prostate samples were cut into $4 \mu \mathrm{m}$-thick histological sections. The samples were placed on the positively charged microscope slides, dried at $37^{\circ} \mathrm{C}$ for $30 \mathrm{~min}$ and incubated at $60^{\circ} \mathrm{C}$ for 60 min using the slide warmer (Premiere, Atlanta, GA, USA). The sections were deparaffinised in Xylene 3 times for $3 \mathrm{~min}$ of each and dehydrated in the alcohol series and tap water. Endogenous peroxidase was blocked by submerging the slides in $0.5 \% \mathrm{H}_{2} \mathrm{O}_{2}$ in methanol for $5 \mathrm{~min}$. Subsequently, the slides were washed in tap water and rinsed in distilled water, heated in TE solution using microwave (Electrolux, Italy) power level 9 for $3 \mathrm{~min}$ and power level 1 for $10 \mathrm{~min}$; and allowed to cool down at room temperature for $30 \mathrm{~min}$. Staining was performed using the Starr Trek Universal HRP Detection System (Biocare, CA, USA) as follows: the slides were placed in PBS pH 7.2 for $5 \mathrm{~min}$, dried and dropped by the protein blocker of background sniper (Biocare) for $15 \mathrm{~min}$ at room temperature and probed with the primary monoclonal antibody of Bcl-2 or Bax (Santa Cruz Biotechnology, CA, USA) 


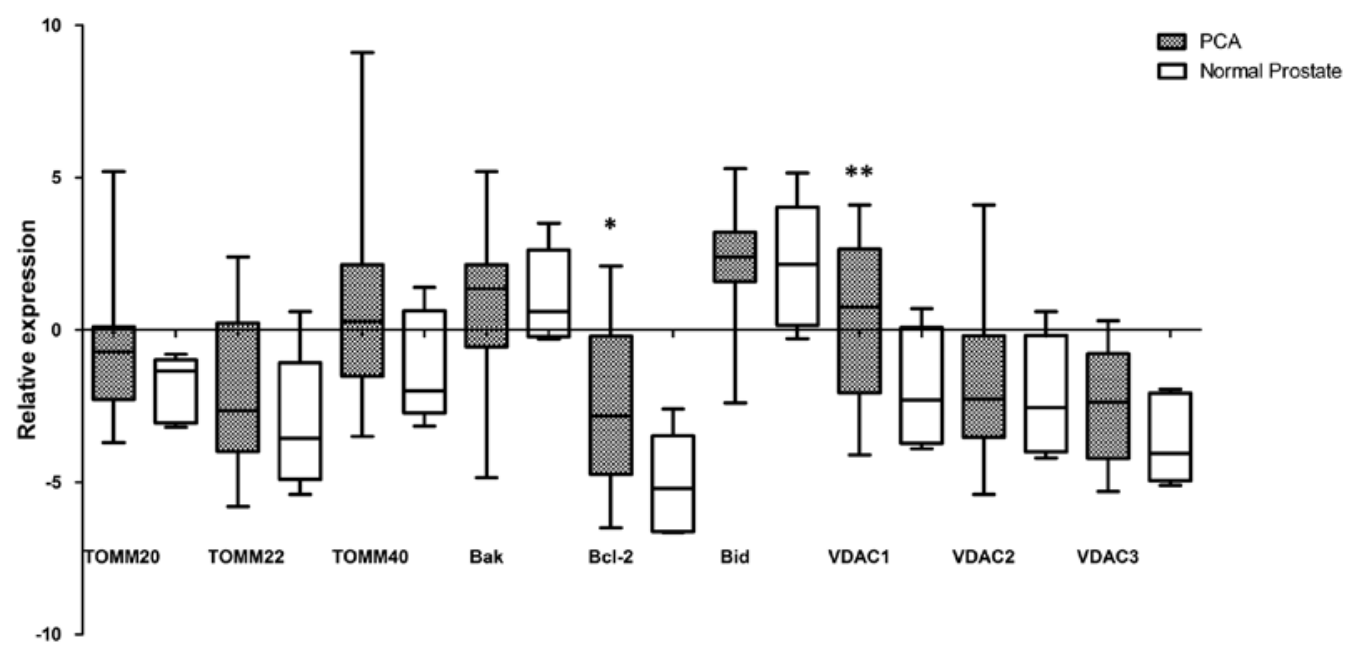

Figure 1. mRNA expression of the TOM20, TOM22 and TOM40, the apoptotic Bak, Bcl-2 and Bid as well as VDAC1, VDAC2 and VDAC3 genes in prostate cancer tissue compared to normal tissue. There were significant differences of the relative expression of Bcl-2 $\left({ }^{*} \mathrm{p}=0.038\right)$ and $\mathrm{VDAC} 1\left(^{* *} \mathrm{p}=0.034\right)$ transcript genes, whereas there were no significant differences of the expression of three isoforms of TOM genes, pro-apoptotic Bak and Bid genes or VDAC2 and VDAC3 genes $(\mathrm{p}>0.05)$ in prostate cancer tissues compared to normal prostate tissues.

or anti-VDAC1 antibody (19). Extensive washing in PBS was carried out before the sections were incubated with Trek Avidin-HRP label (Biocare) for $20 \mathrm{~min}$ at room temperature. The samples were washed again three times. Betazoid diaminobenzidine chromogen (Biocare) was then added to the sections which were incubated in the dark for 5 min before being counter stained in haematoxylin for $60 \mathrm{sec}$, washed in tap water, dehydrated in ascending grades of alcohol before cleaning with xylene and mounting under a cover slip.

Semi-quantitative analysis of expression of Bcl-2, Bax and VDACl antigen in tissue sections. Histological slides of normal prostate or prostate cancers stained with antibodies for Bcl-2 or VDAC1 were digitalized using both the microscope Olympus BX51TR-32FB3F01 with a lamp house for 100W halogen and the camera DP25-SET. The images were digitalized at magnification, $x 1,000$ and snap resolution 2,650 x 1,920. They were saved in the jpg format. The images were analyzed by the semiquantitative algorithm DAB Immune Membrane written as the Image J plugin by Vilppu Tuominen and Jorma Isola of the Cancer Biology Research Group, Institute of Biosciences and Medical Technology (BioMediTech), University of Tampere, Finland. This plugin is a part of the open-source software package (Image J, NIH, USA). The plugin evaluates both intensity and completeness of staining of prostate glands or cell infiltrates. First, it segments diaminobenzidine-stained (DAB) regions of the image. Then, classifies the image based on the staining completeness and intensity. One calibrates the algorithm for the first measurement by defining contrast, intensity range and scale in pixels $/ \mu \mathrm{m}$. Subsequently, the algorithm was tested and tuned up against the images representing both a positive control, i.e., a strong PSA-positive prostate cancer tissue and a negative control, i.e., a PSA-negative colonic mucosa.

Statistical analysis. The number of cycles required for the fluorescent signal to cross the threshold in qPCR method (cycle threshold/Ct) values for each $\mathrm{qPCR}$ product, where the amplification curves corrected against the background crossed a threshold value, were applied in the statistical analysis. Some candidate reference genes were selected using GeNorm software (20). Applying that software, ACTB and HPRT1 were found to be the best combination of two reference genes. For further analysis of the real-time RT-PCR data, we combined ACTB and HPRT1 as the reference genes for normalization of gene expression. Relative expression levels were calculated using the software GenEx Pro 4.3.7 (MultiD Analyses AB Copyright $^{\oplus}$, Weihenstephan, Germany). The mean expression of mRNA corresponding to nine selected genes was analyzed in the group of prostate cancers or in normal prostates using Student's t-test at $\mathrm{p}<0.05$.

\section{Results}

Transcript expressions of mitochondrial-apoptotic molecules (Bak, Bid, Bcl-2), -transport molecules (VDAC1, VDAC2, VDAC3, TOM20, TOM22 and TOM40). Quantitative PCR array demonstrated a significant increase in mRNA expression of anti-apoptotic Bcl-2 gene ( $\mathrm{p}=0.038)$ and VDAC1 gene $(p=0.034)$ in all 14 prostate cancer tissues compared with 5 normal tissue samples. No significant difference in mRNA expression was found between the pro-apoptotic Bak and Bid, VDAC2 and VDAC 3 genes as well as TOMM20, TOMM22, TOMM40 genes in prostate cancer tissue samples and normal tissues ( $p>0.05$ ) (Fig. 1).

Expression of Bcl-2, Bax and VDACl at the protein level in prostate tissues. The expression of Bcl-2, Bax and VDAC1 at the protein level in normal prostate and cancer prostate tissues can be appreciated in Fig. 2: A and B, C and D, E and F, respectively. All three proteins were dispersed in a form of the cytoplasmic grains with a faint membrane staining. In particular, the positive signals of the anti-apoptotic Bcl-2 protein appeared in basal epithelial cells rather than in secretory ones (Fig. 2A). Those proteins were expressed in cells in a relatively broad range as measured by intensity and 

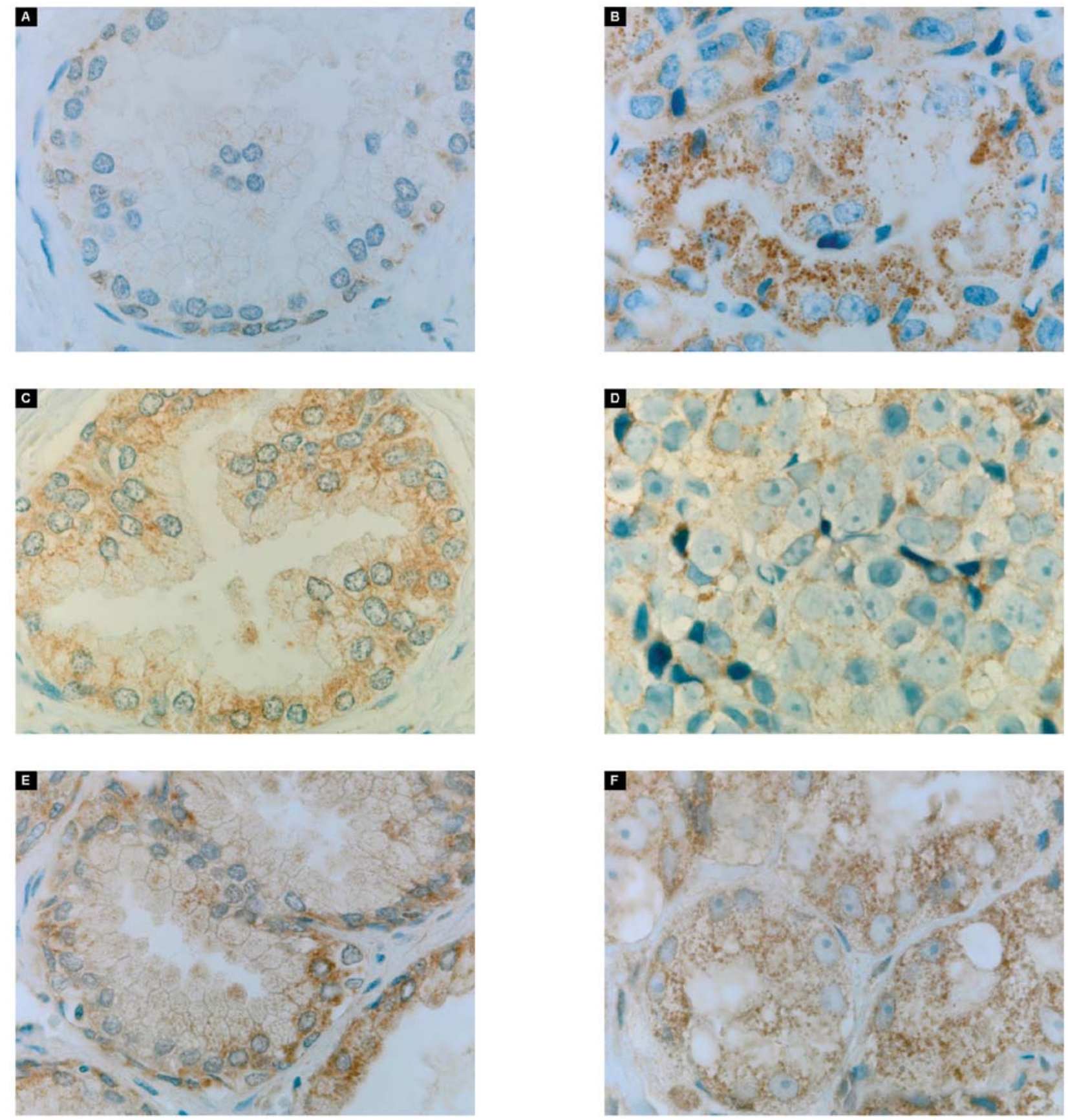

Figure 2. Expression of the anti-apoptotic Bcl-2 protein in the histological section of (A) normal prostate and (B) prostate adenocarcinoma; pro-apoptotic Bax in histological section of (C) normal prostate and (D) prostate adenocarcinoma; VDAC1 protein in histological section of (E) normal prostate and (F) prostate adenocarcinoma. Magnification, x1,000.

completeness, i.e., IM-score (Fig. 3). There was a significant difference in translation of Bax, but not in translation of Bcl-2 and VDAC1 $(\mathrm{p}<0.05, \mathrm{p}=0.887$ and $\mathrm{p}=0.814$, respectively). The semiquantitative algorithm indicates that Bcl-2 and VDAC1 in prostate cancer cells has a tendency to increased expression in comparison with normal prostate. However, this difference is not statistically significant. On the contrary, there was a significant decrease in Bax expression in prostate cancer tissues as compared to normal prostate (Fig. 3).

In the ROC analysis, Bax is shown as the only marker with sufficient statistical power to distinguish between normal prostate (NP) and prostate cancer (PCa) (Fig. 4A). Bax protein sensitivity was $100 \%$ and specificity $64 \%$ at the cut-off value
6.60. The other two markers were not useful producing AUC of about 0.5 or lower (Fig. 4B).

\section{Discussion}

By using PCR array method, this study showed the transcript expression of three kinds of apoptotic proteins of the Bcl-2 family, i.e., pro-apoptotic Bak and Bid, anti-apoptotic Bcl-2 and protein subunits of the outer mitochondrial membrane transport complexes, i.e., three isoforms of voltage dependent anion channels (VDAC1, VDAC2 and VDAC3) as well as three isoforms of the translocase of the outer mitochondrial membrane (TOM20, TOM22 and TOM40) in prostate cancer 


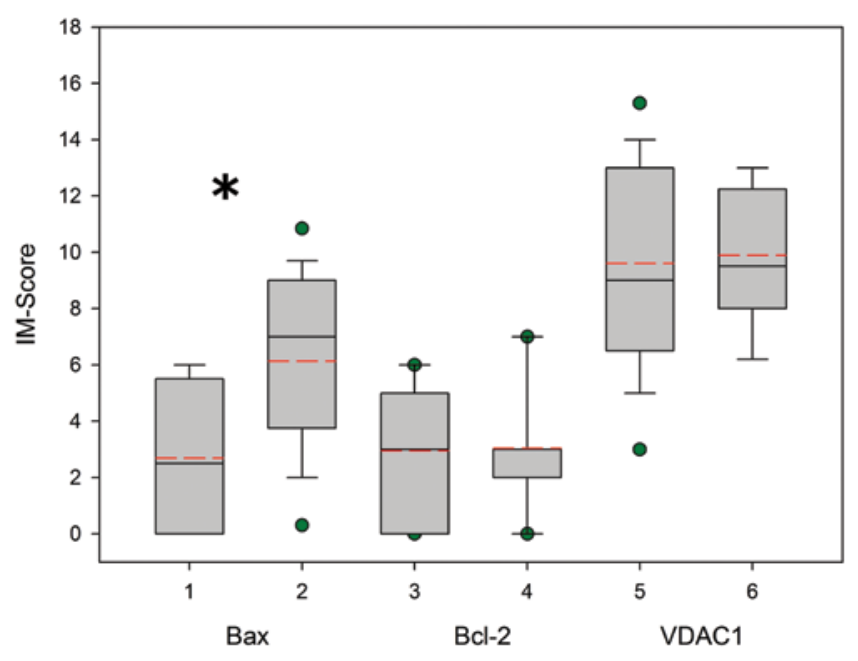

Figure 3. Translation of Bax, Bcl-2 and VDAC1 protein in prostate cancer $(1,3$ or 5 ) or in normal prostate (2, 4 or 6$)$. The green circles represent the $5 \%$ and $95 \%$ percentile. The red dotted line depicts the mean values. The black line represents the median. ${ }^{*} \mathrm{p}<0.05$ denotes significant difference.

tissues compared to normal prostate tissues. We found that the transcript expression of the anti-apoptotic Bcl-2 protein and VDAC1 increased significantly in prostate cancer tissues compared to normal ones. When we compared the transcript expression of Bcl-2 and VDAC1 between normal tissues and prostate cancer with Gleason score $>7$ (10 samples) the increase was even more significant $(\mathrm{p}=0.028)$. Unfortunately, we were not able to analyze the differences of the level expression of both genes in normal tissues and prostate cancer tissues with Gleason score $<7$ due to lack of sufficient amount of samples (4 samples). The transcript expression of VDAC1 might be related to the increased expression of anti-apoptotic
Bcl-2 in cancer cells. Both proteins would inhibit apoptosis of prostate cancer cells.

Hence, we conclude that the increased Bcl-2 mRNA expression is associated with progression of prostate cancer. Our finding is in concert with the previous observations that $\mathrm{Bcl}-2$ gene may be involved in progression of prostate cancer (21). The increased amount of the $\mathrm{Bcl}-2$ transcript in prostate cancer tissue was found also in prostatic intra-epithelial neoplasia. Overexpression of the anti-apoptotic $\mathrm{Bcl}-2$ protein causes effects similar to those of an oncogene (22).

A relationship between gene expression measured as mRNA amount of Bcl-2 as well as VDAC1 and the amount of the appropriate protein is non-linear. The mean amounts of Bcl-2 or VDAC1 mRNA are greater in prostate cancer than in normal prostate. Those differences disappear at the protein level. In addition, translation of the genes in normal prostate does not produce different amounts of the proteins to the extent of that in prostate cancer and results in greater values of standard deviation. Other researchers have found a reverse relationship. For example, Fuzio et al demonstrated that the Bcl-2 mRNA level was significantly decreased in prostate cancer in comparison with normal prostate tissues. Androgen deprivation therapy increased the Bcl-2 mRNA level. However, this difference was not significant (10).

Immunostaining demonstrated in normal prostate tissues positive signals of the anti-apoptotic $\mathrm{Bcl}-2$ protein in basal epithelial cells (Fig. 2A). This finding is in line with the results of Hockenbery et al showing that this anti-apoptotic protein was confined to the basal cells of the prostate epithelium, and was undetectable in secretory cells (23). The disparity between these two cell types is marked by their different capacity to express the anti-apoptotic proteins (20).

Proteins of the Bcl-2 family play a pivotal role in the mitochondrial apoptotic pathway. The ratio between pro-apoptotic and anti-apoptotic molecules determines
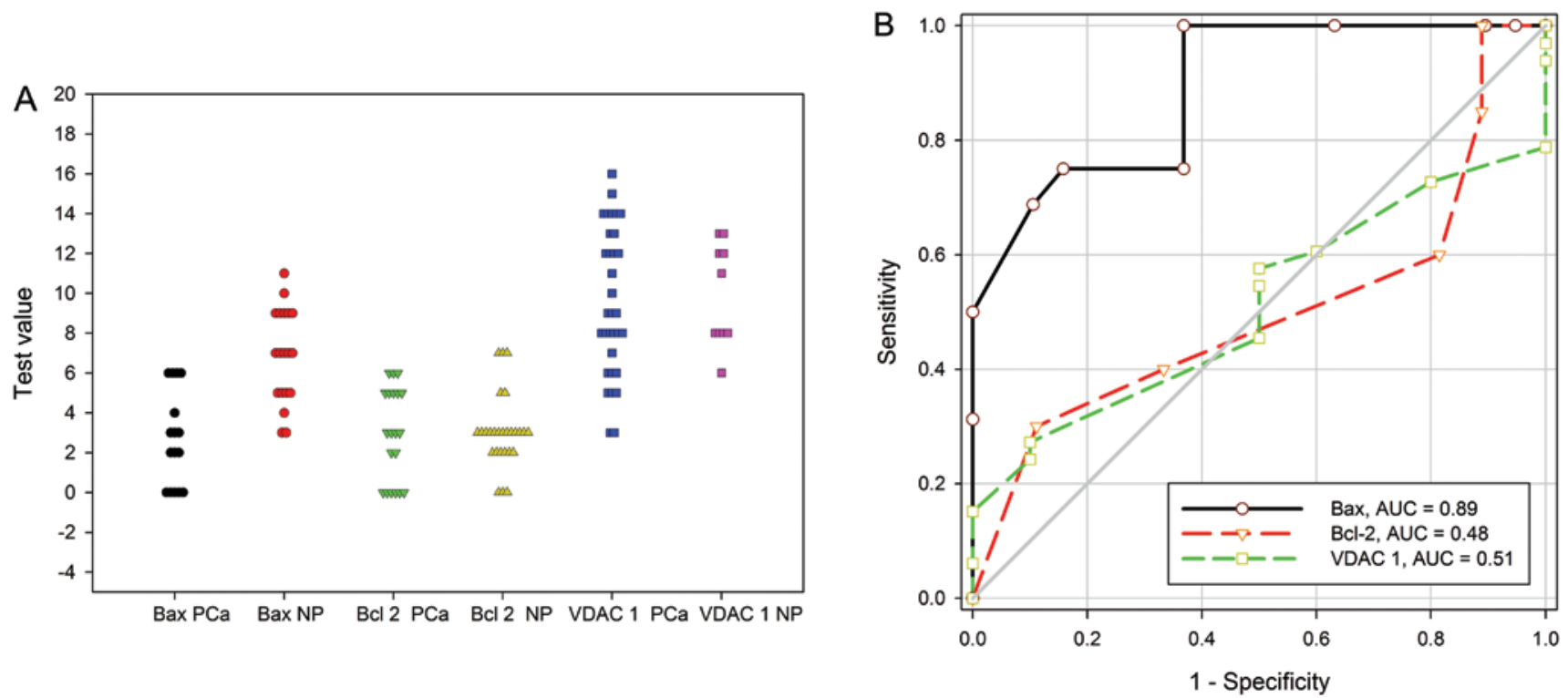

Figure 4. ROC analysis of the expression of Bax, Bcl-2 and VDAC1 protein in prostate cancer (PCa) and normal prostate (NP) presented as (A) a dot histogram and (B) ROC diagram. AUC stands for area under curve. The AUC value for Bax protein is the only value $>0.5$. Sensitivity and specificity for this marker was 100 and $64 \%$, respectively. The cut-off value of the Bax expression that allows separation of normal prostatic tissue from cancer tissue was 6.60 . 
whether or not apoptosis will occur $(5,6)$. In this study, we analyzed the transcript expression of two pro-apoptotic molecules, Bak and Bid, resulting in no significant transcript expression of both genes in prostate cancer tissue in comparison to normal prostate. However, expression of Bax at the protein level decreased significantly in prostate cancer tissues in comparison to normal prostate tissues. Since Bax is the pro-apoptotic protein, one can expect that the decline of its expression be entangled in prostate tumorigenesis. Consequently, the ratio of the anti-apoptotic Bcl-2 to the pro-apoptotic Bax is expected to be increased during tumorigenesis. This study suggests that the high $\mathrm{Bcl}-2 / \mathrm{Bax}$ ratio may contribute to the emergence of prostate cancer by disrupting apoptosis cascade.

Furthermore, the ROC analysis in this study showed the normal prostate and prostate cancer tissue can be separated using Bax in protein level as a marker with sensitivity $100 \%$ at the cut-off value 6.6. However, VDAC1 and $\mathrm{Bcl}-2$ proteins were not informative. These findings suggest the evaluation of Bax in protein level with immunostaining can be used as diagnostic method to determine prostate cancer tissue.

We also evaluated the transcript expression of the mitochondrial transport system, especially in the outer membrane that mediates the cytochrome $c$ release in order to initiate apoptosis. There are at least two main transport systems in the outer mitochondrial membrane that co-operate with Bcl-2 family proteins, porin, known also as voltage dependent anion channel (VDAC) and the translocase of the outer mitochondrial membrane (TOM) complex (24). It has been reported that $\mathrm{Bax} / \mathrm{Bak}$ and $\mathrm{Bcl}-\mathrm{xL}$, but not $\mathrm{Bik}$ and $\mathrm{Bid}$, can bind directly to the voltage-dependent anion channel (VDAC) and modulate its activity to induce apoptosis in mammalian cells $(14,15)$. It was shown that the $\mathrm{N}$-terminal region of VDAC1 isoform structurally interacts with the Bcl-2 and Bcl-xL to mediate anti-apoptotic effects $(12,16)$. The immunoblot study with isolated rat liver mitochondria was performed to elucidate which receptor could bind to the pro-apoptotic Bax. This study showed that Bax was associated with TOM complexes, especially TOM22. Furthermore, this analysis demonstrated that Bax could not associate with VDAC1. It was suggested that formation of the mitochondrial permeability pore was involved in the interaction between Bax and TOM22 while triggering apoptosis (17). Working with isolated mitochondria from yeast, Ott et al demonstrated that TOM40 played a role in the Bax translocation into the outer mitochondrial membrane and its action to release cytochrome $c$ (18). However, Ross et al described that there was no direct dependence of Bax and Bak insertion and oligomerization in the outer mitochondrial membrane on the import and assembly machineries of TOM in HeLa cells (25). The use of different cell types in these studies is most likely responsible for these differences. Indeed, apoptosis in yeast and mammalian cells depends on different molecular events. For example, yeast lacks Bcl-2like proteins. Although we did not analyze the interaction between the family of Bcl-2 proteins, i.e. the pro-apoptotic Bak and Bid as well as the anti-apoptotic Bcl-2 and proteins in the mitochondrial transport complexes, i.e., VDAC and TOM isoforms, we could show that there was no significant differences in the expression of pro-apoptotic Bak and Bid or
TOMM20, TOMM22 and TOMM40 transcripts in prostate cells with the altered apoptotic process. This finding suggests that the activities of the TOM complexes do not play any essential role in development of prostate cancer.

Furthermore, we found a significant difference in transcript expression of VDAC1 isoform in prostate cancer cells compared to normal prostate cells, but not in VDAC2 and VDAC3 expressions. As a channel protein, VDAC is located not only in the mitochondrial membrane of eukaryotes but also in the extra-mitochondrial regions of eukaryotic cells, e.g., in the plasmalemma (26-29). In mammals there are three isoforms of VDAC protein. The distribution, characterization and function of the three isoforms of VDAC differ and the transcript level of VDAC isoforms varies in different tissues and species (reviewed in ref. 13). In higher eukaryotes, VDAC1 is the most predominant isoform. Several studies demonstrated differences in the expression levels of VDACs in cancer cells and normal cells, most of them showed alteration in VDAC1 expression (30). In a study with 11 human cancer cell lines, Simamura et al reported that cancer cells expressed higher levels of VDAC1 than normal cells such as WI-38 fibroblasts (31). The study, combining affinity labeling of cell surface molecules, avidin affinity chromatography and mass spectrometry analyses of cell membrane proteins of normal and cancerous cells originating from one prostate cancer patient, revealed reduced expression level for type-1 (VDAC1) and type-2 (VDAC2) porins in the mitochondrial membrane of cancer cells (32). Several studies demonstrated VDAC1 upregulation in various cancer cell lines, which is induced by different treatments, i.e., in three acute lymphoblastic leukemia (ALL) cell lines after prednisolone treatment (33), in cervix squamous cell carcinoma cell line (A431) after cisplatin treatment (34), and in human malignant melanoma cell line (A375) after treatment with tyrosinase inhibitor arbutin (35). In prostate cancer cell lines, it has been reported that the VDAC1 expression in $\mathrm{LNCaP}$ cell line was approximately two times higher than its expression in PC-3 and DU 145 cell lines. Increased expression in prostate cancer cell lines was also induced by G3139, the 18 -meric phosphorothionate antisense oligonucleotide; an inducer of caspase-dependent apoptosis (36). Since cells cultured in vitro represent a supramolecular system pre-selected in very specific conditions, their profile of gene expression will not overlap with the profile of cells living in vivo. Hence, the conclusive power of such analyses is limited, and their results may not reflect the biological reality. Looking from that perspective, our results seem to be more realistic for tissue systems, such as prostate cancer.

The VDAC1 was also proposed to be involved in the extrinsic apoptotic pathway. This channel appears to interact with different modulators such as sigma-1 receptor and estrogen receptor ER $\alpha$ in the cell membrane. For example, it can induce the extrinsic apoptotic pathway in LNCaP cell line (37). For the reasons stated above, it is unknown if this effect takes place in prostatic tissue in vivo.

We demonstrated the level of expression and the localization of VDAC1 in prostate cancer tissues (Fig. 2F) and normal prostate tissues (Fig. 2E). The strong positive signal was found in both basal epithelial and secretory cells of normal prostate gland. This finding denotes that both cell types need 
this protein for regulation of both apoptosis and cell growth. Indeed, VDAC plays an important role in energy production via controlling metabolite traffic across the outer mitochondrial membrane (38). It has been reported that the downregulation of VDAC1 expression would result in decreased energy production and, thus, may affect cell vitality. The decrease in hVDAC1 levels resulted in a dramatic decrease in cell growth, a 5-fold decrease in ATP synthesis, as well as a decrease of about $50 \%$ in the cellular levels of ATP and ADP (39). The channel of VDAC regulates ATP flux from mitochondria (40). The closure state of VDAC channel shows a higher permeability to $\mathrm{Ca}^{2+}$, reduces metabolite flux, and thus sensitizes mitochondria to apoptotic signals (41). The positive signal of VDAC1 expression was found mainly in secretory cells of the prostate cancer gland.

Our study demonstrates that VDAC1, not TOM complex, together with the anti-apoptotic protein, Bcl-2 plays a role in prostate tumorigenesis. However, it remains unclear how VDAC1 is involved in development of prostate cancer. The level of interference may not primarily be gene expression but posttranslational complex assembly, since in the context of apoptosis VDAC may be induced to form the oligomeric complexes. Of course, this is the case with the composition of TOM isoforms. Expression of the protein subunits is one aspect, but formation of the complexes that would be active in the physiological, impaired or pathological way is the other one. Pro- or antiapoptotic proteins of the Bcl-2 family may interfere with either the level of biosynthesis, gene expression and complex assembly. This issue remains beyond the scope of this article.

\section{Acknowledgements}

This study was supported by International Collaborative Research Fund from University of Indonesia. The authors are grateful to Dr Dwi Ari Pujianto, Faculty of Medicine University of Indonesia, for his help in editing the manuscript.

\section{References}

1. Adams JM: Ways of dying: multiple pathways to apoptosis. Genes Dev 17: 2481-2495, 2003.

2. Thompson CB: Apoptosis in the pathogenesis and treatment of disease. Science 267: 1456-1462, 1995.

3. Bidere N, Su HC and Lenardo MJ: Genetic disorders of programmed cell death in the immune system. Annu Rev Immunol 24: 321-352, 2006.

4. Danial NN and Korsmeyer SJ: Cell death: critical control points. Cell 116: 205-219, 2004.

5. Wong RBY: Apoptosis in cancer: from pathogenesis to treatment. J Exp Clin Cancer Res 30: 87, 2011.

6. Ola MS, Nawaz M and Ahsan H: Role of Bcl-2 family proteins and caspases in the regulation of apoptosis. Mol Cell Biochem 351: 41-58, 2011.

7. McDonnell TJ, Troncoso P, Brisbay SM, Logothetis C, Chung LW, Hsieh JT, et al: Expression of the protooncogene BCL-2 in the prostate and its association with emergence of androgenindependent prostate cancer. Cancer Res 52: 6940-6944, 1992.

8. Raffo AJ, Perlman H, Chen MW, Day ML, Streitman JS and Buttyan R: Overexpression of BCL-2 protects prostate cancer cells from apoptosis in vitro and confers resistance to androgen depletion in vivo. Cancer Res 55: 4438-4445, 1995.

9. McConkey DJ, Greene G and Pettaway CA: Apoptosis resistance increase with metastatic potential in cells of the human LNCaP prostate carcinoma line. Cancer Res 56: 5594-5599, 1996.

10. Fuzio P, Ditonno P, Lucarelli G, Battaglia M, Bettocchi C, Senia T and Perlino E: Androgen deprivation therapy affects BCL-2 expression in human prostate cancer. Int J Oncol 39: $1233-1242,2011$.
11. Yoshino T, Shiina H, Urakami, S, Kikuno N, Yoneda T, Shigeno K and Igawa M: Bcl-2 expression as a predictive marker of hormone-refractory prostate cancer treated with taxane-based chemotherapy. Clin Cancer Res 12: 6116-6124, 2006.

12. Geula S, Ben-hail D and Shoshan-Barmartz V: Structure-based analysis of VDAC1: N-terminus location, translocation, channel gating and association with anti-apoptotic protein. Biochem $\mathbf{J}$ 444: 475-485, 2012.

13. Messina A, Reina S, Guarino F and De Pinto V: VDAC isoforms in mammals. Biochim Biophys Act 1818: 1466-1476, 2012.

14. Shimizu S, Matsuoka Y, Shinohara Y, Yoneda Y and Tsujimoto Y: Essential role of voltage-dependent anion channel in various forms of apoptosis in mammalian cells. J Cell Biol 152: 237-250, 2001.

15. Shore GC: Apoptosis: it's Bak to VDAC. EMBO Rep 10: 1311-1313, 2009.

16. Abu-Hamad S, Arbel N, Calo D, Arzoine L, Israelson A, Keinan N, Ben-Romano R, Friedman O and ShoshanBarmatz V: The VDAC1 N-terminus is essential both for apoptosis and the protective effect of anti-apoptotic proteins. J Cell Sci 122: 1906-1916, 2009.

17. Bellot G, Cartron PF, Er E, Oliver L, Juin P, Armstrong LC, Bornstein P, Mihara K, Manon S and Vallette FM: TOM22, a core component of the mitochondria outer membrane protein translocation pore, is a mitochondrial receptor for the proapoptotic protein Bax. Cell Death Differ 14: 785-794, 2007.

18. Ott M, Norberg E, Zhivotovsky B and Orrenius S: Mitochondrial targeting of tBid/Bax: a role for the TOM complex? Cell Death Differ 16: 1075-1078, 2009.

19. Cassará MC, Menzel VA, Hinsch KD, Wrenzycki C and Hinsch E: Voltage-dependent anion channels 1 and 2 are expressed in porcine oocytes. Biosci Rep 30: 193-200, 2009.

20. Vandesompele J, De Preter K, Pattyn F, Poppe B, Van Roy N, De Paepe A and Speleman F: Accurate normalization of the real-time quantitative RT-PCR data by geometric averaging of multiple internal control genes. Genome Biol 3: RESEARCH0034, 2002.

21. Catz SD and Johnson JL: Bcl-2 in prostate cancer: a minireview. Apoptosis 8: 29-37, 2003.

22. Lemelynova AA, Grygorenko VM, Cheremuha SV and Romanenko AM: Correlation between histological type and immunohistochemical profile of prostate cancer and Gleason scale gradation. J Exp Oncol 31: 246-249, 2009.

23. Hockenbery DM, Zutter M, Hickey W, Nahm M and Korsmeyer SJ: Bcl2 protein is topographically restricted in tissues characterized by apoptotic cell death. Proc Natl Acad Sci USA 88: 6961-6965, 1991 .

24. Motz C, Martin H, Krimmer T and Rassow J: Bcl-2 and porin follow different pathways of TOM-dependent insertion into the mitochondrial outer membrane. J Mol Biol 323: 729-738, 2002.

25. Ross K, Rudel T and Kozjak-Pavlovic V: TOM-independent complex formation of Bax and Bak in mammalian mitochondria during TNFalpha-induced apoptosis. Cell Death Differ 16: 697-707, 2009.

26. Schein SJ, Colombini $M$ and Finkelstein A: Reconstitution in planar lipid bilayers of a voltage-dependent anion-selective channel obtained from Paramecium mitochondria. J Membr Biol 30: 99-120, 1976.

27. Benz R: Permeation of hydrophilic solutes through mitochondrial outer membranes: review on mitochondrial porins. Biochim Biophys Acta 1197: 167-196, 1994.

28. Thinnes FP: Evidence for extra-mitochondrial localization of the VDAC/porin channel in eukaryotic cells. J Bioenerg Biomembr 24: 71-75, 1992

29. De Pinto V, Messina A, Lane DJ and Lawen A: Voltagedependent anion-selective channel (VDAC) in the plasma membrane. FEBS Lett 584: 1793-1799, 2010.

30. Shoshan-Barmatz V and Mizrachi D: VDAC1: from structure to cancer therapy. Front Oncol 2: 164, 2012.

31. Simamura E, Hirai K, Shimada H, Koyama J, Niwa Y and Shimizu S: Furanonaphthoquinones cause apoptosis of cancer cells by inducing the production of reactive oxygen species by the mitochondrial voltage-dependent anion channel. Cancer Biol Ther 5: 1523-1529, 2006.

32. Hastie C, Saxton M, Akpan A, Cramer R, Master JR and Naaby-Hansen S: Combined affinity labeling and mass spectrometry analysis of differential cell surface protein expression in normal and prostate cancer cells. Oncogene 24: 5905-5913, 2005.

33. Jiang N, Kham SK, Koh GS, Suang Lim JY, Ariffin H, Chew FT and Yeoh AE: Identification of prognostic protein biomarkers in childhood acute lymphoblastic leukemia (ALL). J Proteomics 74: 843-857, 2011 
34. Castagna A, Antonioli P, Astner H, Hamdan M, Righetti SC, Perego P, Zunino F and Righetti PG: A proteomic approach to cisplatin resistance in the cervix squamous cell carcinoma cell line A431. Proteomics 4: 3246-3267, 2004.

35. Nawarak J, Huang-Liu R, Kao SH, Liao HH, Sinchaikul S, Chen ST and Cheng SL: Proteomic analysis of A375 human malignant melanoma cells in response to arbutin treatment. Biochim Biophys Acta 1792: 159-167, 2009.

36. Lai JC, Tan W, Benimetskaya L, Miller P, Colombini M and Stein CA: A pharmacologic target of G3139 in melanoma cells may be the mitochondrial VDAC. Proc Natl Acad Sci USA 103: 7494-7499, 2006.

37. Thinnes FP: Neuroendocrine differentiation of LNcaP cells suggests: VDAC in the cell membrane is involved in the extrinsic apoptotic pathway. Mol Genet Metab 97: 241-243, 2009.
38. Shoshan-Barmatz V, Keinan $\mathrm{N}$ and Zaid $\mathrm{H}$ : Uncovering the role of VDAC in the regulation of cell life and death. J Bioenerg Biomembr 40: 183-191, 2008

39. Abu-Hamad S, Sivan S and Shoshan-Barmatz V: The expression level of the voltage-dependent anion channel controls life and death of the cell. Proc Natl Acad Sci USA 103: 5787-5792, 2006.

40. Rostovtseva T and Colombini M: VDAC channels mediate and gate the flow of ATP: implications for the regulation of mitochondrial function. Biophys J 72: 1954-1962, 1997.

41. Tan W, Colombini M: VDAC closure increases calcium ion flux. Biochim Biophys Acta 1768: 2510-2515, 2007. 